doi: ISSN 0103-5150

Fisioter. Mov., Curitiba, v. 26, n. 4, p. página 725-741, set./dez. 2013

Licenciado sob uma Licença Creative Commons

\title{
Auditoria em fisioterapia no Sistema Único de Saúde: proposta de um protocolo específico
}

\author{
Audit in physiotherapy in the Unified Health \\ System: proposal of specific protocol
}

\author{
Ítalo Ricardo Santos Aleluia ${ }^{[a]}$, Fabiane Costa Santos ${ }^{[b]}$
}

[a] Fisioterapeuta, mestrando em Saúde Coletiva pela Universidade Federal da Bahia (ISC/UFBA) e membro do Programa Integrado de Cooperação Técnica em Formação e Avaliação de Atenção Básica (GRAB-ISC-UFBA), Salvador, BA, Brasil, e-mail: italoaleluia@yahoo.com.br.

[b] Fisioterapeuta, mestranda em Saúde Coletiva pela Universidade Federal da Bahia (ISC/UFBA), professora especialista da Faculdade Social da Bahia (FSBA) e Faculdade de Tecnologia e Ciências de Salvador (FTC), Salvador, BA, Brasil, e-mail: ptfabiane@yahoo.com.br.

\section{Resumo}

Introdução: A auditoria no Sistema Único de Saúde (SUS) zela pela eficácia, eficiência e efetividade dos serviços que integram o sistema. Nos serviços de fisioterapia, as auditorias têm sido desempenhadas por profissionais sem os conhecimentos técnicos da área e inexistem protocolos de auditoria em fisioterapia específicos para o SUS, o que tem representado um entrave para a especificidade e qualidade das auditorias fisioterapêuticas realizadas. Objetivo: Este estudo teve como objetivo propor um modelo de protocolo específico de auditoria fisioterapêutica para os serviços públicos de fisioterapia. Materiais e métodos: O presente trabalho trata-se de uma pesquisa exploratória por meio do levantamento de artigos publicados nas bases de dados LILACS, SciELO e portal Latin Science, além de documentos eletrônicos do Ministério da Saúde, Coffito e Associação Portuguesa de Fisioterapeutas, entre outros, entre 1991 a 2012. Respeitando as informações colhidas, o protocolo construído contempla uma auditoria sistemática e global sobre os mais variados aspectos dos serviços de fisioterapia em suas dimensões normativas, estruturais, processuais, bem como de resultados. Resultados: Espera-se que o protocolo construído possa contribuir para aumentar a 
eficiência e acurácia da auditoria em fisioterapia no SUS, de modo que isso se traduza em melhorias na qualidade dos serviços prestados aos usuários.

Palavras-chave: Gestão. Auditoria. Sistema Único de Saúde. Fisioterapia.

\section{Abstract}

Introduction: The audit in the SUS, watches over for the effective, efficiency and effectiveness of the services that system integrate. In the physiotherapy services, the audit have been performed by professionals without the technician knowledge in the field and inexist specific protocols of audit in physiotherapy to the SUS, has represented important obstacle to the specificity of physiotherapy audit carried through. Objective: This study it had as objective to propose a model of specific protocol of Physiotherapy Audit. Materials and methods: The present work this was an exploratory research, surveyed published in the databases LILACS, SciELO and Latin Science portal, and electronic documents of the Coffito, Health of Ministry and Portuguese Association of Physiotherapy between the years of 1991 to 2011. Respecting the information provided in articles and documents selected, the protocol constructed search to contemplate a broad and systematic audit on the most varied aspects of the physiotherapy services, in their normative dimensions, structural, procedural and results. Results: It is expects that the constructed protocol can contribute to increase the efficiency an accuracy of the physiotherapy audit in SUS, so this is translated the improvement quality of services provided to the users.

Keywords: Management. Audit. Unified Health System. Physiotherapy.

\section{Introdução}

A auditoria no Sistema Único de Saúde (SUS) consiste em ações de verificação sistemática que utilizam a medição, a observação e o ensaio de uma atividade, elemento ou sistema, a fim de comprovar a adequação dos serviços às leis e normas existentes, na perspectiva de determinar se as ações de saúde, bem como seus resultados, estão em conformidade com o planejado. Tal atividade tem sido crucial como ferramenta de avaliação e controle dos serviços públicos de saúde, já que sua finalidade principal é garantir ou melhorar a qualidade desses serviços e viabilizar a otimização de recursos $(1,2,3)$.

A importância da auditoria no SUS passou a ser enfatizada desde a criação das Leis Orgânicas da Saúde (LOS), em 1990, que estabeleceram a necessidade da criação de um Sistema Nacional de Auditoria (SNA) desdobrado nos três níveis de gestão. Entretanto, a criação desse sistema ocorreu apenas por meio da Lei n. 8.689, de 27 de julho de 1993, que, posteriormente, foi regulamentada pelo Decreto n. 1.651, de 28 de setembro de $1995(4,5,6,7)$.

Dentre os objetivos da auditoria no SUS estão os de controlar a execução dos serviços de saúde, detectar situações que exijam aprofundamento, avaliar a estrutura, os processos aplicados e os resultados alcançados, aferindo sua adequação aos critérios e parâmetros de eficiência, eficácia e efetividade, tendo como atributo final proporcionar aos usuários do sistema melhor equilíbrio entre os recursos aplicados e a qualidade dos serviços prestados $(6,7,8)$.

0 processo de trabalho na auditoria do SUS tem sido desempenhado por equipes multiprofissionais, em que médicos, enfermeiros, nutricionistas, odontólogos e farmacêuticos já conquistaram seu espaço e desempenham suas funções mediante integração de conhecimentos e experiências $(9,10)$.

Isso corrobora com a visão do SNA, que defende o trabalho da auditoria no SUS desempenhado por um grupo multiprofissional como um requisito para a eficiência e a resolutividade da atividade por meio da integração de profissionais relacionados aos mais variados serviços que integram a rotina do sistema (2).

Apesar disso, alguns estudos têm demonstrado o predomínio de atuação de médicos e enfermeiros nas equipes do setor público, em que ainda não existem evidências de participação dos profissionais de fisioterapia $(9,10,11)$.

Tal situação faz com que os serviços de fisioterapia sejam auditados por profissionais sem os conhecimentos técnicos dessa área, e isso, talvez, dificulte a 
especificidade e qualidade da auditoria fisioterapêutica no SUS, repercutindo em fragilidades na gestão dos recursos e qualidade nesses serviços $(12,13,14)$.

Estudos têm apontado dificuldades dos auditores de saúde na auditagem de serviços que não integram sua área de conhecimento, em decorrência da falta de conhecimentos técnicos e por esta exigir muito do conhecimento específico de cada área da saúde. Inclusive, os próprios auditores enfatizaram a carência de outros profissionais nas equipes, a exemplo dos fisioterapeutas $(6,7,15)$.

Especificamente em fisioterapia, o trabalho de Aleluia (11) demonstrou diversas dificuldades dos auditores do SUS que atuam nos serviços de fisioterapia na Bahia. Tais dificuldades tiveram uma relação direta com a falta de conhecimentos técnicos e experiência dos auditores acerca dos procedimentos, equipamentos, organização e funcionamento dos serviços, com implicações importantes no desempenho das auditorias fisioterapêuticas.

Apesar dessa situação, os serviços públicos de fisioterapia continuam carecendo de uma atenção maior em termos de especificidade na auditoria, pois ainda não existem protocolos ou roteiros que possam sistematizar e aumentar a eficiência da atividade (11).

Dessa forma, tal realidade tem colocado as equipes do SNA em situações de escassez de instrumentos, parâmetros e indicadores de avaliação e recomendações, o que possivelmente repercute em entraves para a tomada de decisões que impactem positivamente na qualidade dos serviços públicos de fisioterapia.

Somado a isso, trabalhos demonstraram que os serviços de fisioterapia no SUS, em todo o Brasil, tem apresentado significativo crescimento na demanda por atendimento e custos para o sistema em todas as especialidades. Para os autores, isso traduz a necessidade da implementação de um controle maior e mais específico nas auditorias fisioterapêuticas, que proporcione à população melhor qualidade na oferta dos serviços de fisioterapia $(12,13,14)$.

Desse modo, é possível que a ausência de instrumentos de auditoria em fisioterapia para o SUS, atrelada à falta de conhecimentos técnicos dos auditores, represente prejuízos para o controle e avaliação do equilíbrio entre os recursos aplicados, qualidade dos serviços e a satisfação final do usuário do sistema.

Logo, a construção de uma proposta de protocolo específico de auditoria em fisioterapia para o SUS pode contribuir para aumentar a eficiência das equipes de auditoria em saúde pública diante dos serviços de fisioterapia, que possa fortalecer o processo de trabalho do SNA e corroborar com um controle mais criterioso sobre os recursos e qualidade desses serviços prestados à população.

Assim, este trabalho teve como objetivo propor um modelo de protocolo específico de auditoria em fisioterapia para os serviços próprios, contratados ou conveniados com o SUS.

\section{Metodologia}

Trata-se de uma pesquisa de caráter exploratório, na qual a construção do protocolo teve como fundamentos teóricos artigos, manuais de normas de auditoria do MS, portarias estaduais e federais de saúde, além de leis e resoluções do Coffito e documentos da Associação Portuguesa de Fisioterapeutas (APF).

Inicialmente foi realizado um levantamento de artigos relevantes e relacionados ao tema publicados nas bases de dados LILACS, SciELO e portal Latin Science e documentos eletrônicos, incluindo legislações e manuais de normas de auditoria, disponíveis nos sites do MS e Coffito. 0 período de corte para o estudo foi de 1991 a 2011, tendo em vista a escassez de trabalhos sobre auditoria de saúde, instrumentos de auditoria e publicações na área de auditoria em fisioterapia, o que inclusive foi considerado como uma limitação para essa pesquisa.

A seleção dos artigos e documentos teve como critérios de inclusão a abordagem sobre: questões relacionadas à avaliação de serviços de saúde e, em alguns destes, itens de verificação de serviços fisioterapêuticos; verificações normativas; de recursos materiais; de recursos humanos; de organização e funcionamento; de prontuários; de gestão de resultados e satisfação de usuários em serviços de saúde. Foram excluídos os documentos não relacionados com os temas descritos.

Considerando esses critérios de inclusão foram selecionados 12 artigos (Paim (16, 17); Campos (18); Mascarenhas (19, 20); Costa (21); Stephan et al. (22); Schieseri et al. (23); Cerchiari et al. (24); Neto et al. (25); Donabeddian (26); Azevedo (27)), sete manuais do MS $(1,28,29,30,31,32,33)$, uma portaria estadual (PT n. 2.101/90 (34)), uma lei estadual (Lei n. 3.982/1981 (35)), duas portarias federais (PTMS n. 33/98 (36), n. 929/2002 (37)), dois documentos da APF (Bettencourt (38); Guerra et al. (39)) e seis dispositivos legais do Coffito (Lei n. 8.856/94 (40), 
Resoluções n. 387/2011 (41), n. 354/2008 (42), n. $242 / 2002$ (43), n. $241 / 2002$ (44), n. $139 / 1993$ (45)), além de dados quantitativos de sessões, códigos e procedimentos de assistência fisioterapêutica disponíveis no Sistema de Gerenciamento da Tabela de Procedimentos, Órteses, Próteses e Materiais Especiais do SUS (SIGTAP) (46).

Tendo em vista o material pesquisado, o protocolo foi subdividido em etapas de acordo com os quesitos de verificação, visando proporcionar ao usuário do instrumento uma auditoria sistemática e mais completa possível diante dos serviços de fisioterapia próprios, contratados ou conveniados do SUS.

Assim, o protocolo consta de uma fase inicial com a identificação do profissional auditor, do tipo, data e motivo desencadeante da auditoria, bem como da identificação do serviço auditado.

Nas demais fases, o protocolo foi categorizado em oito blocos com questões específicas cada um, tais como: bloco I (verificação normativa), bloco II (verificação da estrutura física), bloco III (verificação dos recursos materiais), bloco IV (verificação dos recursos humanos), bloco $\mathrm{V}$ (verificação da organização e funcionamento do serviço), bloco VI (verificação dos prontuários), bloco VII (verificação da gestão de resultados) e bloco VIII (verificação da satisfação dos usuários).

0 mecanismo de avaliação dos respectivos blocos consiste na marcação de respostas a cada uma das perguntas que compõem o bloco avaliado. As respostas disponíveis foram subdivididas em: "Sim", "Não" e “ Não se Aplica (N/A)". Desse modo, o auditor marcará apenas uma resposta em conformidade com aquilo que foi identificado no serviço de fisioterapia, e escolherá o item N/A toda vez que a pergunta de algum bloco não tiver correlação com o regime do serviço auditado, quais sejam: hospitalar, ambulatorial ou domiciliar.

\section{Resultados}

Respeitando as informações colhidas nos artigos e documentos selecionados, buscou-se a construção de um protocolo (Anexo 1) que abrangesse o máximo de quesitos relevantes nos serviços de fisioterapia por meio de uma abordagem de controle e avaliação global, a fim de proporcionar uma visão ampla sobre os aspectos mais relevantes da avaliação em saúde, tais como: estrutura, processo e resultados.

A proposta do protocolo consiste em uma fase inicial constituída da identificação de itens importantes durante o processo de auditagem. Esses itens foram sugeridos com a finalidade de registrar informações que possibilitem uma reauditoria com a melhor confiabilidade possível, em virtude da necessidade de reavaliar o impacto que as modificações e recomendações pós-auditoria tiveram sobre a qualidade do serviço.

No bloco I foi proposta uma verificação de itens relacionados aos aspectos legais e burocráticos de rotina dos serviços de fisioterapia essenciais para o funcionamento dos estabelecimentos, incluindo avaliação do atendimento às disposições legais do Coffito, Vigilância Sanitária e Ministério da Saúde (MS).

O bloco II permite uma verificação da estrutura física dos serviços, que inclui aspectos de acessibilidade aos portadores de necessidades especiais, higiene, adequação de metas e objetivos com a política do serviço, capacidade tecnológica, segurança e conforto do ambiente, existência de espaços adequados para a execução das avaliações, técnicas e acolhimento dos usuários.

Considerando a grande variedade e a importância de manter o adequado funcionamento dos equipamentos e materiais disponíveis nos serviços de fisioterapia, os itens do bloco III apontam para a gestão dos recursos materiais, incluindo a existência de equipamentos mínimos determinados pelo MS e para substituição emergencial, licença e registro dos equipamentos e materiais na Anvisa, comprovação de manutenções preventivas e corretivas, existência de equipamentos danificados ou desativados, quantitativos de recursos materiais, adequação tecnológica e sua relação com o atendimento à demanda, bem como condições de armazenamento.

A verificação dos recursos humanos também foi enfatizada no protocolo, já que os profissionais fazem parte do processo de trabalho e apresentam estreita relação com a qualidade na prestação dos cuidados aos usuários dos serviços. 0 bloco IV aborda principalmente questões relacionadas ao cadastramento e atualização dos profissionais no CNES; obediência à jornada de trabalho estabelecida em lei e seu respectivo cumprimento, bem como discrepâncias e compatibilidade de carga horária dos profissionais com a produtividade do serviço; avaliação de regularidade de registro no órgão de classe; evidência de exercício ilegal da profissão por estagiários ou técnicos de fisioterapia; existência de políticas de capacitação continuada e medidas de prevenção dos riscos ocupacionais, assim como disponibilidade e utilização de Equipamentos de Proteção Individual (EPIs) necessários e participação dos profissionais 
nas avaliações, planejamento e tomada de decisões no serviço.

A verificação da organização e funcionamento dos serviços (bloco V) tem como objetivo compreender de que forma os serviços têm sido planejados para protocolar o atendimento aos usuários; evitar ou reduzir filas; agilizar o processo de atendimento; manter todos os encaminhamentos e alta registrados; avaliar sua capacidade instalada; normatizar suas rotinas frente aos colaboradores e gerenciar suas metas no atendimento, comparando o planejado com o executado; existência de mecanismos de humanização no atendimento; existência de fluxos de referência e contrarreferência; relação entre os parâmetros assistenciais nacionais e adequação da produtividade, entre outros.

No bloco VI foi proposta a verificação de quesitos em prontuários, tendo em vista a importância desse instrumento para avaliação da qualidade na assistência prestada. Os pontos a serem analisados nos prontuários são: se de fato existem; se são informatizados e se estão corretamente armazenados; se suas descrições estão legíveis e coerentes; se contêm itens essenciais da avaliação clínica do exame físico, bem como o diagnóstico cinésico-funcional; se existe descrição detalhada das condutas e objetivos; se apresentam quantitativo de sessões prescritas e sua adequação aos códigos dos procedimentos vigentes na tabela do SUS; se as evoluções estão sendo feitas diariamente ou a cada sessão; se existe cumprimento de prazo e registro das reavaliações; se existem rasuras, numeração das páginas do prontuário, carimbo e assinatura do profissional responsável pela execução do atendimento ao final das evoluções; e se há respeito ao limite máximo de sessões em regime ambulatorial e hospitalar conforme o SIGTAP.

O bloco de verificação da gestão de resultados (bloco VII) tem como propósito analisar se os serviços em questão possuem estratégias de gestão baseadas em resultados para melhoria e aperfeiçoamento de suas falhas, visando aumentar a qualidade e a satisfação dos usuários. Logo, esse bloco contempla a verificação de espaços de escuta para os colaboradores e usuários, avaliação do desempenho da equipe e satisfação de seus colaboradores, indicadores de atendimentos resolutivos, atendimento e resolutividade para com as reclamações e sugestões, utilização de indicadores para tomada de decisões, além do controle de cobertura e acesso.

Por fim, o bloco VIII foi direcionado para um dos atributos mais importantes de mensuração da qualidade em saúde: os usuários. Desse modo, essa etapa é constituída de perguntas que visam encontrar discrepâncias ou concordâncias entre as informações encontradas pelo auditor nos itens anteriores, com a real impressão e satisfação final do usuário que utiliza ou utilizou o serviço.

\section{Discussão}

Atualmente, para o SUS, o único documento que retrata alguns quesitos de auditoria em fisioterapia traz uma visão pouco específica, generalista e que contempla apenas verificações do processo assistencial e carga horária dos fisioterapeutas, dispensando itens de verificação normativa, de recursos materiais, humanos, processo de funcionamento, entre outros (2). Tal documento, apesar de ser o mais utilizado pelas equipes de auditoria do SUS no que tange aos serviços de fisioterapia, apresenta-se como uma ferramenta frágil, de pouca acurácia e que não dispõe de indicadores que verifiquem as mais variadas dimensões dos serviços fisioterapêuticos referentes à estrutura, processo, gestão de resultados e satisfação dos usuários. É possível que isso implique em dificuldades na mensuração da conformidade dos serviços.

Apesar disso, Bettencourt (35) ressalta que durante o processo de auditoria em fisioterapia deve-se dar importância à identificação de todos os problemas e necessidades em diversos aspectos desses serviços, formulados com base nas informações obtidas por meio das avaliações subjetivas e objetivas.

Autores destacam a imprescindibilidade de pilares como a estrutura, o processo e os resultados durante a auditoria operacional em saúde como indicadores relacionados à relevância, eficiência e efetividade dos resultados proporcionados pelos serviços de saúde $(6,8,47)$.

Segundo Gil et al. citado por Almeida (48), com relação aos aspectos de avaliação em saúde, os serviços de fisioterapia ocupam destaque devido ao crescimento da necessidade de assistência por essa profissão, evidenciando a importância da avaliação dos mais variados atributos de eficiência, efetividade e eficácia.

Estudos avaliaram os gastos e a demanda por atendimento de fisioterapia no SUS e encontraram resultados que demonstram o comportamento crescente dos custos e o quantitativo de sessões com esse serviço no Brasil, impactando no consumo de 
parcelas significativas do financiamento público da saúde. Em virtude dessa realidade, os autores destacaram a necessidade de um controle maior e mais específico sobre os serviços públicos de fisioterapia que proporcione melhor qualidade na prestação desse serviço à população $(12,13,14)$.

Ainda como outro fator relevante, de acordo com o Cadastro Nacional dos Estabelecimentos de Saúde (CNES), estima-se que, atualmente, existem no Brasil cerca de 1.781.000 (um milhão, setecentos e oitenta e um mil) serviços de fisioterapia integrando o SUS, apesar de todas as questões relacionadas à alimentação e sub-registros desse sistema (49).

Esses são motivos mais que suficientes para justificar a necessidade de auditorias mais criteriosas e específicas, sistematizadas por meio de instrumentos que aumentem a acurácia e eficiência da auditoria nos serviços de fisioterapia que integram o SUS.

No entanto, no trabalho de Aleluia (11), quando identificados os dificultadores do processo de auditoria nos serviços de fisioterapia no SUS, na Bahia, os depoimentos dos auditores que atuam no estado colocaram como um dos entraves para o controle e avaliação eficaz a inexistência de roteiros ou protocolos de auditoria em fisioterapia para o SUS. Tal fato tem colocado as equipes do SNA em escassez de parâmetros para tomada de decisões, com base em indicadores concretos e seguros que possam impactar em melhorias qualitativas sobre os serviços públicos de fisioterapia.

Para Silva (50), a importância de medir é incontestável. O autor ressalta a importância do setor público dispor de ferramentas de gestão que englobem parâmetros ou indicadores de eficiência e eficácia. Para ele, somente por meio da mensuração são tomadas medidas corretivas, oportunas e adequadas, evitando o processo decisório com base no casuísmo ou na mera intuição.

Segundo Cruz (1999) citado por Silva (50), sem controle os rumos não são corrigidos, os objetivos fundamentais ficam colocados em segundo plano, há desperdício e inadequação no uso dos recursos.

Stephan (22) e Scarparo (51) atentam para a escassez de práticas de avaliação nos serviços de saúde. Ambos enfatizam a importância da construção de instrumentos e metodologias para o exercício da auditoria que contemplem os objetivos de acordo com a estrutura, processo e resultado da assistência, definindo o caminho a percorrer para o alcance da qualidade.

O controle em saúde, baseado na avaliação da gestão por meio de indicadores, representa uma ferramenta fundamental para o desenvolvimento e fortalecimento da administração pública e da qualidade dos serviços prestados, além do grau de satisfação dos usuários (50).

Stephan (22) afirma que a implementação de um processo de avaliação dos serviços de saúde, de forma sistemática sobre estruturas, processos e resultados, permite o melhor planejamento e compreensão dos problemas para as ações de controle e auditora, contribuindo para aumento da qualidade, eficiência, eficácia e efetividade dos serviços. Isso de fato é o que se pretende com a proposta do protocolo de auditoria fisioterapêutica em questão.

De acordo com Mascarenhas (2000) citado por Soares (52), é imprescindível que todas as organizações de saúde construam instrumentos de auditoria. Isso possibilita padronizar o processo de trabalho de seus diversos auditores, servindo como ferramenta de garantia, principalmente do exercício profissional do auditor.

Considerando as dificuldades amplamente comentadas na literatura, no que tange à falta de conhecimentos técnicos de auditores para com serviços que não integram a sua formação profissional (a exemplo dos serviços de fisioterapia) e a inexistência de protocolos de auditoria fisioterapêutica para o SUS, a proposta deste instrumento pretende contribuir para fortalecer o trabalho das equipes de auditoria em saúde pública. Portanto, é necessário melhorar a efetividade e acurácia do SNA sobre o controle e avaliação dos serviços de fisioterapia, de forma que suas ações possam impactar nesses serviços em termos de melhorias na qualidade, eficiência e resolutividade, aumentando a satisfação dos usuários do sistema.

\section{Conclusão}

O controle e a avaliação dos serviços de saúde por meio de auditoria são pontos cruciais para a garantia do equilíbrio entre os recursos, a qualidade da assistência e a satisfação dos usuários.

Entretanto, os serviços de fisioterapia que integram o SUS, embora apresentem significativo crescimento na produtividade e custos para o sistema público de saúde, com um imenso quantitativo de estabelecimentos no Brasil, carecem de roteiros e protocolos específicos que propiciem uma auditoria mais criteriosa e específica, já que isso dificulta o trabalho 
da equipe de auditoria que, praticamente em todo o Brasil, não conta com profissionais fisioterapeutas.

Dessa forma, espera-se que o protocolo proposto possa corresponder às necessidades da equipe e auxiliar na sistematização do processo de auditoria de fisioterapia no SUS por meio de um controle mais específico e global, com enfoque nas dimensões estruturais, do processo de trabalho, funcionamento e resultados propiciados aos usuários, visando contribuir para a gestão eficaz sobre os recursos e qualidade dos serviços próprios, contratados ou conveniados, e que tenha como objetivo final aumentar a satisfação da população.

Faz-se necessário salientar que a proposta deste protocolo não reduz a importância da construção de novos instrumentos nem a relevância da participação do fisioterapeuta nas equipes de auditoria.

Novos estudos que possam contribuir com informações relevantes para a gestão dos serviços públicos de fisioterapia são importantes, principalmente por poderem esclarecer pontos fundamentais da auditoria fisioterapêutica.

\section{Referências}

1. Ministério da Saúde (Brasil). Departamento de controle, avaliação e auditoria. Manual normas de auditoria. Brasília: Ministério da Saúde; 1998.

2. Ministério da Saúde (Brasil). Departamento Nacional de Auditoria do SUS. Auditoria no SUS: noções básicas sobre sistemas de informação. 2. ed. Brasília: Ministério da Saúde; 2004.

3. Noronha F, Salles M. A ferramenta auditoria aplicada à odontologia. In: Anais do 1. Congreso Virtual Brasileño de Administración; 14-17 out 2004. [acesso 20 nov 2013]. Disponível em: http://www.convibra.com. br/artigo.asp?ev=25\&id=1860

4. Brasil. Lei n. 8.689, de 27 de julho de 1993. Dispõe sobre a extinção do Instituto Nacional de Assistência Médica da Previdência Social (INAMPS) e dá outras providências. Brasília, DF; 1993. [acesso em 5 nov 2013]. Disponível em: http://www.planalto.gov.br/ ccivil_03/leis/L8689.htm

5. Brasil. Decreto n. 1.651, de 28 de setembro de 1995. Regulamenta o Sistema Nacional de Auditoria do Sistema Único de Saúde. Brasília, DF; 1995. [acesso em 5 nov 2013]. Disponível em: http://www.planalto.gov. br/ccivil_03/decreto/1995/d1651.htm
6. Melo MB. O Sistema Nacional de Auditoria do SUS: estruturação, avanços, desafios e força de trabalho [dissertação]. Rio de Janeiro: Escola Nacional de Saúde Pública Sérgio Arouca, Fundação Oswaldo Cruz; 2006.

7. Souza J. A prática da auditoria de enfermagem no Estado de Santa Catarina: a percepção dos auditores a partir da análise do discurso do sujeito coletivo [dissertação]. Florianópolis: Universidade do Oeste de Santa Catarina; 2006.

8. Melo M, Vaitsman J. Auditoria e avaliação no Sistema Único de Saúde. São Paulo perspect. 2008;22(1):152-64.

9. Pimentel LR. O trabalho do auditor do SUS: facilidades, dificuldades, avanços e desafios. Revista Sul Americana de Auditoria em Saúde. 2008;1(2):241-84.

10. Pinto K. A prática da enfermeira em auditoria em saúde [dissertação]. Salvador: Universidade Federal da Bahia; 2005.

11. Aleluia IRS. Principais dificuldades encontradas pelos auditores do Sistema Único de Saúde nos serviços de fisioterapia do Estado da Bahia [monografia de especialização]. Salvador: Universidade Estácio de Sá; 2012.

12. Santos FC, Aleluia IRS, Santos IN, Moura LGF, Carvalho MA. Participação do fisioterapeuta na equipe multiprofissional de auditoria em saúde. Rev adm saúde. 2011;13(51):95-102.

13. Santos FC, Sady CM, Costa ES. Participação do fisioterapeuta na auditoria em saúde. Fisioter Bras. 2010;11(3):226-31.

14. Santos FC. Auditoria de sistemas e serviços de saúde: participação do fisioterapeuta. Revista Sul Americana de Auditoria em Saúde. 2008;1(2):423-33.

15. Middleton S, Middleton P G. Fisioterapia para problemas respiratórios e cardíacos. 2. ed. Rio de Janeiro: Gunabara Koogan; 1994.

16. Paim CRP, Zucchi P. Auditoria de avaliação dos serviços de saúde no processo de credenciamento. Ciênc saúde coletiva. 2011;16(supl. 1):1163-71.

17. Paim CRP, Ciconelli RM. Auditoria de avaliação da qualidade em serviços de saúde. Rev adm saúde. 2007; 9 (36):85-92. 
18. Campos JF, Souza SROS, Saurusaitis AD. Auditoria de prontuários: avaliação dos registros de aspiração traqueal em terapia intensiva. Rev Eletr Enf. 2008;10(2):358-66.

19. Mascarenhas EB. Registro de prontuários em fisioterapia: instrumento de auditoria. Revista Sul Americana de Auditoria em Saúde. 2010;3(1):97-122.

20. Mascarenhas EB. Auditoria de qualidade em fisioterapia: um instrumento para medir a satisfação do usuário. Revista Sul Americana de Auditoria em Saúde. 2010;3(1):69-96.

21. Dias-da-Costa JS, Madeira ACC, Luz RM, Britto MAP. Auditoria médica: programa de pré-natal em posto de saúde na região Sul do Brasil. Rev Saúde Pública. 2000;34(4):329-36.

22. Stephan AMS, Dias-da-Costa JS, Hoefel AL. Auditoria médica: atendimento de crianças com asma em uma Unidade de Saúde da Família, Pelotas, RS. Rev bras epidemiol. 2009;12(2):172-9.

23. Schieseri LMC, Kissil M. Avaliação da qualidade nos hospitais brasileiros. Rev adm saúde. 2003;5(18):7-17.

24. Cerchiari GSF, Erdmann RH. Sistema de informações para acompanhamento, controle e auditoria em saúde pública. RAP. 2008;42(5):925-48.

25. Neto DC, Silva IO, Costa ES. Dimensões da auditoria em saúde. Revista Sul Americana de Auditoria em Saúde. 2009;2(2):133-48.

26. Donabeddian A. Evaluation de la calidad de la atención médica. In: White KL, editor. Investigaciones, sobre servicios de salud: uma antologia. Washington: Organización Panamericana de la Salud; 1992. p. 383-404.

27. Azevedo A. Avaliação do desempenho dos serviços de saúde. Rev Saúde Pública. 1991;25(1):65-71.

28. Ministério da Saúde (Brasil). Departamento de Regulação Avaliação e Controle. Manual de auditoria técnico científica. 2. ed. Brasília: Ministério da Saúde; 1999. [acesso 10 abr 2011]. Disponível em: http://www. sgc.goias.gov.br/upload/links/arq_780_arq_865_ManualAdeAAuditoriaATecnicoACientifica.pdf

29. Ministério da Saúde (Brasil). Departamento de Regulação Avaliação e Controle. Programa Nacional de Avaliação do Serviços de Saúde (PNASS). Brasília: Ministério da Saúde; 2004. [acesso 10 abr 2011]. Disponível em: http:// www.anvisa.gov.br/servicosaude/avalia/PNASS.pdf
30. Ministério da Saúde (Brasil). Departamento Nacional de Auditoria do SUS. Orientações Técnicas sobre Auditoria na Assistência Ambulatorial e Hospitalar do SUS. Brasília: Ministério da Saúde; 2005. [acesso 14 abr 2010]. Disponível em: http://bvsms.saude.gov.br/bvs/publicacoes/ auditoria_assistencia_ambulatorial_hospitalar_v3.pdf

31. Ministério da Saúde (Brasil). Departamento de Regulação Avaliação e Controle. Manual técnico do Cadastro Nacional dos Estabelecimentos de Saúde. 2 ed. Brasília: Ministério da Saúde; 2006. [acesso 14 set 2011]. Disponível em: http://dtr2001.saude.gov.br/ sas/download/manual\%20cnes\%20atualizado\%20 em\%2010_11_06\%2015\%20h.pdf

32. Ministério da Saúde (Brasil), Departamento de Regulação Avaliação e Controle. Manual do Sistema de Informação Ambulatorial (SIA). Brasília: Ministério da Saúde, 2008. [acesso 10 abr 2011]. Disponível em: http://portal.saude.gov.br/portal/arquivos/pdf/MANUAL\%20SISAIH01_AGOSTO\%202008.pdf

33. Ministério da Saúde (Brasil). Departamento de Regulação Avaliação e Controle. Manual do Sistema de Informação Hospitalar (SIH). Brasília: Ministério da Saúde; 2009. [acesso 10 abr 2011]. Disponível em: http://w3.datasus. gov.br/sihd/Manuais/Manual_SIH_Junho_2009.pdf

34. Bahia. Secretaria de Saúde do Estado. Portaria Estadual n. 2.101, de outubro de 1990. Estabelece normas de Vigilância Sanitária. Coletânea de legislação básica em Vigilância Sanitária. v. 1. Salvador: Governo da Bahia; 1998. [acesso 6 nov 2013]. Disponível em: http://www1.saude.ba.gov.br/divisa/arquivos/mat-publico/manual-legislacao-basica.pdf

35. Bahia. Lei n. 3.982, de 29 de dezembro de 1981. Dispõe sobre o subsistema de saúde do Estado da Bahia, aprova a legislação básica sobre a promoção, proteção e recuperação da saúde e dá outras providências. Coletânea de legislação básica em Vigilância Sanitária. v. 1. Salvador: Governo da Bahia; 1998. [acesso 6 nov 2013]. Disponível em: http://www1.saude.ba.gov.br/divisa/arquivos/ mat-publico/manual-legislacao-basica.pdf

36. Brasil. Ministério da Saúde. Portaria n. 33, de 24 de março de 1998. Publica o modelo padronizado da Ficha Cadastral de Estabelecimentos de Saúde - FCES Módulos Básicos, bem como o respectivo manual de instruções. Diário Oficial [da] República Federativa do Brasil, 25 mar 1998; Seção 1. p. 42. 
37. Brasil. Ministério da Saúde. Portaria n. 929, de 26 de novembro de 2002. Determina que, para o estabelecimento de saúde proceder à cessão de crédito a pessoas jurídicas ou físicas, conceituadas como estabelecimentos de saúde, pela PT/SAS/ 11, de 29 de dezembro de 2000, estas deverão igualmente estar cadastradas no CNES e o estabelecimento que a contrata deverá reconhecê-la como seu serviço de terceiros, informando no campo serviços especializados da FCES a condição de terceiros e indicando o seu CNPJ/CPF. Brasília, DF, 2002. [acesso 6 nov 2002]. Disponível em: http://dtr2001.saude.gov.br/sas/PORTARIAS/ PORT2002/PT-929.htm

38. Bettencourt C, Machado IO, Fonseca JP, Pascoalinho J, Avillez M, Murta 0, et al. Instrumentos de auditoria aos padrões da prática. São Domingos da Rana: Associação Portuguesa de Fisioterapeutas; 2005. [acesso 6 nov 2013]. Disponível em: http://www.apfisio.pt/ Ficheiros/InstAuditoria.pdf

39. Isabel de Souza Guerra IS, Bettencourt C, Fonseca JP, Pascoalinho J, Avilez M, Murta O. Normas de boas práticas para as unidades de fisioterapia. São Domingos da Rana: Associação Portuguesa de Fisioterapeutas; 2008. [acesso 6 nov 2013]. Disponível em: http: http://apfisio.pt/PadroesPraticas/NormasUnidFisioterapia.pdf

40. Brasil. Lei n. 8.856, de 1 de março de 1994. Fixa a jornada de trabalho dos profissionais Fisioterapeuta e Terapeuta Ocupacional. Brasília, DF; 1994. [acesso 8 out 2011]. Disponível em: http://www.planalto.gov. br/ccivil_03/Leis/L8856.htm

41. Conselho Federal de Fisioterapia e Terapia Ocupacional - Coffito. Resolução n. 387, de 8 de junho de 2011. Fixa e estabelece parâmetros assistenciais fisioterapêuticos nas diversas modalidades prestadas pelo fisioterapeuta e dá outras providências. [acesso 8 out 2011]. Disponível em: http://www.coffito.org.br/publicacoes/pub_view. asp? $\operatorname{cod}=2034 \&$ psecao $=9$

42. Conselho Federal de Fisioterapia e Terapia Ocupacional - Coffito. Resolução n. 354, de 8 de novembro de 2008. Dispõe sobre a Licença Temporária de Trabalho (LTT), para os fins a que destina e dá outras providências. [acesso em 8 out 2011]. Disponível em: http://www.coffito.org.br/publicacoes/pub_view. asp?cod $=1618 \&$ psecao $=9$
43. Conselho Federal de Fisioterapia e Terapia Ocupacional - Coffito. Resolução n. 242, de 7 de novembro de 2002. Dispõe sobre o veto ao registro no COFFITO de título de tecnólogo em Fisioterapia e ao exercício da atividade ao seu portador. [acesso em 8 out 2011]. Disponível em: http://www.coffito.org.br/publicacoes/pub_view.asp?cod $=1324 \&$ psecao $=9$

44. Conselho Federal de Fisioterapia e Terapia Ocupacional - Coffito. Resolução n. 139, de 28 de novembro de 1993. Dispõe sobre as atribuições do exercício da responsabilidade técnica nos campos assistenciais da Fisioterapia e Terapia Ocupacional. [acesso em 8 out 2011]. Disponível em: http://www.coffito.org.br/publicacoes/pub_view. asp?cod $=1066 \&$ psecao $=9$

45. Conselho Federal de Fisioterapia e Terapia Ocupacional - Coffito. Resolução n. 241, de 23 de maio de 2002. Dispõe sobre o exercício ilegal de atividade regulamentada por portadores de certificados de técnico em reabilitação e/ou em Fisioterapia e dá outras providências. [acesso em 8 out 2011]. Disponível em: http://www.coffito.org.br/publicacoes/pub_view. asp?cod $=1323 \&$ psecao $=9$

46. Ministério da Saúde (Brasil). Sistema de Gerenciamento da Tabela de Procedimentos, Órteses, Próteses e Materiais Especiais (SIGTAP). Grupo: procedimentos clínicos. Subgrupo: Fisioterapia. [acesso 12 set 2011]. Disponível em: http://sigtap. datasus.gov.br/tabela-unificada/app/sec/relatorio/ procedimentoAtributos/publicados

47. Caleman G, Moreira ML, Sanchez MC. Auditoria, controle e programação de serviços de saúde. São Paulo: Fundação Petrópolis; 1998.

48. Almeida DCS. Ragasson CAP, Lazzarotto EM, Salmoria JG. A gerência na ótica do Fisioterapeuta. In: Anais do 2. Seminário Nacional de Estado e Políticas Sociais no Brasil; 13-15 out 2005; Cascavel. Cascavel: Unioeste; 2005.

49. Ministério da Saúde (Brasil). Cadastro Nacional dos Estabelecimentos de Saúde CNES: consulta a serviços especializados. Fisioterapia-Bahia. [acesso 14 nov 2011]. Disponível em: http://cnes.datasus.gov. br/Lista_Es_Municipio_Mantenedora.asp?VEstado= 29\&VCodMunicipio=292740\&NomeEstado=BAHIA \&NomeMun=SALVADOR 
50. Silva ARL. Auditoria: uma ferramenta social. Portal da educação: gestão e liderança. 2008 [23 abr 2009]. Disponível em: http://www.portaleducacao.com.br/gestao-e-lideranca/artigos/3841/ auditoria-uma-ferramenta-social

51. Scarparo AF, Ferraz CA. Auditoria em enfermagem: identificando sua concepção e métodos. Rev bras enferm. 2008;61(3):302-5.

52. Soares PRB. O perfil do médico auditor das Unimed do Rio Grande do Sul [monografia]. Porto Alegre: Universidade Gama Filho; 2001.

Recebido: 15/04/2012

Received: 04/15/2012

Aprovado: $21 / 05 / 2013$

Approved: 05/21/2013 
Anexo 1

\section{PROTOCOLO DE AUDITORIA EM FISIOTERAPIA}

\section{IDENTIFICAÇÃO DO AUDITOR}

Nome:

Categoria profissional:

Registro profissional:

Esfera: ( ) Municipal ( ) Estadual $\quad($ ) Federal

\section{IDENTIFICAÇÃO DA AUDITORIA}

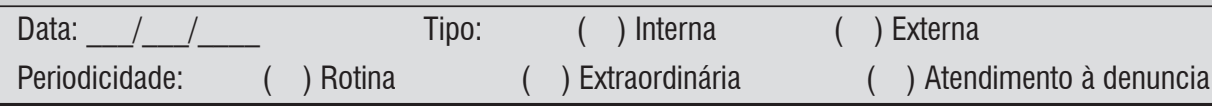

\section{IDENTIFICAÇÃO DO SERVIÇO}

Município:

Nome da unidade:

Endereço:

CEP:

Bairro:

Serviço: $\quad$ ( ) Próprio

Regime:

Prestador:
( ) Ambulatorial

( ) Municipal

( ) Estadual

\section{Estado:}

CNES No:

Complemento:

\section{( ) Contratado}

( ) Hospitalar

( ) Privado

( ) Universitário
( ) Conveniado

( ) Domiciliar

( ) Filantrópico

( ) Federal

\section{Bloco I - VERIFICAÇÃO NORMATIVA}

O serviço em questão possui registro de firma em cartório?

0 serviço em questão possui alvará de funcionamento?

0 alvará de funcionamento encontra-se em local de fácil visibilidade?

0 alvará de funcionamento apresenta prazo de validade regular?

0 serviço possui alvará sanitário emitido pelo órgão competente?

0 alvará sanitário encontra-se em local de fácil visibilidade?

0 alvará sanitário apresenta prazo de validade regular?

0 serviço possui registro no órgão de classe competente?

0 serviço possui um responsável técnico legalmente habilitado?

0 responsável técnico é um fisioterapeuta em obediência à Resolução Coffito n.139/93?

0 responsável técnico encontra-se no estabelecimento durante 0 expediente de funcionamento?

Percebe-se que o serviço executa ações que the cabem legalmente?

Os anúncios ou formas de propaganda do estabelecimento encontram-se em conformidade com a Portaria n. 1.201/90?

A unidade possui um Plano de Gerenciamento de Resíduos Sólidos de Serviços de Saúde (PGRSS)? 


\section{BIoco I - VERIFICAÇÃO NORMATIVA}

0 PGRSS está sendo colocado em prática?

0 estabelecimento está devidamente cadastrado no CNES?

0 cadastramento do estabelecimento no CNES está devidamente atualizado?

Todas as informações básicas sobre o serviço estão disponíveis no CNES?

Em caso de serviço contratado o estabelecimento apresentou número de contrato com o SUS?

\begin{tabular}{|l|l|l|}
\hline Sim & Não & N / A \\
\hline & & \\
\hline & & \\
\hline & & \\
\hline & & \\
\hline
\end{tabular}

\section{Bloco II - VERIFICAÇÃO DA ESTRUTURA FÍSICA}

0 serviço possui localização que facilita 0 acesso dos usuários?

0 serviço dispõe de declaração e descrição de metas e objetivos que refletem as necessidades e interesses dos indivíduos que atende?

0 serviço tem capacidade para realizar os procedimentos cadastrados?

0 serviço apresenta boas condições físicas e tecnológicas?

0 serviço adota métodos que visam garantir a segurança e a integridade física dos usuários?

0 serviço dispõe de ambientes como sala de espera ou recepção?

Existe um espaço exclusivo destinado à administração do serviço?

Existem salas exclusivas para exames e avaliações fisioterapêuticas?

0 serviço é composto de espaços e ambientes adequados ao tratamento e aplicação de técnicas?

Os sanitários são separados por sexo e distante dos ambientes comuns?

Os sanitários são adaptados para portadores de necessidades especiais?

0 serviço dispõe de sanitários exclusivos para os colaboradores?

0 serviço dispõe de pia com água corrente, piso antiderrapante de fácil limpeza e higienização?

As paredes do estabelecimento são de cor clara, lisa, impermeável e de fácil higienização?

0 ambiente em geral possui boas condições de higienização?

As portas de acesso possuem dimensões mínimas de 0,80 m de largura, conforme Portaria $\mathrm{n}$. $1.201 / 90$, e são de fácil manejo?

0 ambiente apresenta barreiras arquitetônicas que inviabilizam 0 acesso de pessoas com necessidades especiais?

Percebe-se que 0 ambiente em geral oferece condições de acessibilidade aos portadores de necessidades especiais?

0 ambiente apresenta temperatura agradável?

A unidade divulga à sociedade seus serviços e ações de forma a reforçar sua imagem e facilitar 0 acesso?

\section{Bloco III - VERIFICAÇÃO DOS RECURSOS MATERIAIS}

serviço dispõe dos equipamentos mínimos determinados na Portaria estadual n. 2.101/90?

0 serviço possui a listagem e a organização de todos os equipamentos por setor?

Os materiais e equipamentos possuem licença ou registro da Anvisa?

Os recursos materiais e equipamentos estão em boas condições de uso?

0 serviço dispõe de documento ou registro que comprova a manutenção corretiva dos recursos materiais e equipamentos? 


\section{BIoco III - VERIFICAÇÃO DOS RECURSOS MATERIAIS}

Existe manutenção preventiva dos materiais e equipamentos?

Existem materiais ou equipamentos com problemas e que necessitam de manutenção ou troca?

Existem equipamentos ociosos ou desativados?

0 quantitativo de recursos materiais atende à demanda de usuários do serviço?

Há disponibilidade de materiais ou equipamentos para atender a necessidade de substituição emergencial?

0 serviço recebe investimentos para os recursos materiais?

Os materiais e equipamentos são compatíveis com a finalidade e técnicas empregadas no serviço?

0 serviço dispõe de todos os manuais dos equipamentos utilizados para esclarecimentos ou instruções?

0 estabelecimento apresenta condições de armazenamento adequada e segura dos recursos materiais e equipamentos?

Percebe-se que os recursos materiais e equipamentos do serviço acompanham a evolução tecnológica da fisioterapia?

\section{Bloco IV - VERIFICAÇÃO DOS RECURSOS HUMANOS}

Todos os profissionais que atuam no serviço estão cadastrados no CNES? Os profissionais do serviço cadastrados no CNES de fato cumprem a sua jornada regularmente?

Existe compatibilidade da carga horária dos profissionais declarada no CNES com a produtividade do serviço?

Existe discrepância de carga horária de algum profissional do respectivo serviço cadastrado no CNES? Os profissionais exercem sua atividade no serviço respeitando a carga horária máxima estabelecida pela Lei n. 8.856/94?

Todos os profissionais que atuam no serviço apresentaram a cédula de registro no órgão de classe competente?

Em caso de profissionais com Licença Temporária de Trabalho (LTT), foi comprovado que seu prazo de validade está regular?

Foi presenciado o exercício das atividades de fisioterapia por estagiários?

Os estagiários presentes no serviço estavam sob supervisão de um professor devidamente vinculado a uma Instituição de Ensino Superior (IES)?

0 serviço possui documento que comprove 0 acompanhamento efetivo do estagiário por um professor de IES conveniada ou supervisor da parte cedente?

Os estagiários são contabilizados como recursos humanos existentes para planejamento da programação de atendimento do serviço?

Foi evidenciado o exercício das atividades exclusivas do fisioterapeuta por técnicos de fisioterapia ou equivalente em desobediência à Resolução n. 242/2002?

0 quantitativo de recursos humanos é suficiente para atender à demanda do serviço?

Existem protocolos de normatização das atividades profissionais?

Existe uma política de educação continuada para os profissionais vinculados ao serviço?

0 serviço mantém registro de atividades de desenvolvimento de educação continuada?

Existe um modelo de recrutamento e seleção adotado pelo serviço?

A equipe de fisioterapeutas é adequada aos serviços que devem ser prestados?

Há equilíbrio entre a equipe e a prestação de um serviço seguro e efetivo?

Existem medidas de gestão e avaliação de riscos ocupacionais para os colaboradores?

0 serviço dispõe dos Equipamentos de Proteção Individual (EPIs) necessários?

\begin{tabular}{l|l|l|l}
\hline Sim & Não & N / A \\
\hline & & \\
\hline & & & \\
\hline & & \\
\hline & & & \\
\hline & & & \\
\hline & & & \\
\hline
\end{tabular}

\begin{tabular}{|c|c|c|}
\hline Sim & Não & $\mathrm{N} / \mathrm{A}$ \\
\hline & & \\
\hline & & \\
\hline & & \\
\hline & & \\
\hline & & \\
\hline & & \\
\hline & & \\
\hline & & \\
\hline & & \\
\hline & & \\
\hline & & \\
\hline & & \\
\hline & & \\
\hline & & \\
\hline & & \\
\hline & & \\
\hline & & \\
\hline & & \\
\hline & & \\
\hline & & \\
\hline
\end{tabular}




\begin{tabular}{l|l|l|l}
\hline Bloco IV - VERIFICAÇÃo DOS RECURSOS HUMANOS & Sim & Não & N / A \\
\hline & & & \\
\hline Os colaboradores utilizam regular e adequadamente os EPIs necessários? & & & \\
\hline Os funcionários são avaliados regularmente por equipe de Medicina do Trabalho? & & & \\
\hline Os postos de trabalho atendem à finalidade e à necessidade do serviço? & & & \\
\hline Os profissionais sabem manusear todos os equipamentos e materiais disponíveis na unidade? & & & \\
\hline Os colaboradores participam do planejamento e avaliação das atividades do serviço? & & \\
\hline
\end{tabular}

\begin{tabular}{|c|c|c|c|}
\hline \multicolumn{4}{|l|}{ Bloco V - VERIFICAÇÃO DA ORGANIZAÇÃO E FUNCIONAMENTO } \\
\hline \multirow[b]{2}{*}{0 serviço dispõe de protocolos de assistência para os usuários? } & Sim & Não & $\mathrm{N} / \mathrm{A}$ \\
\hline & & & \\
\hline \multicolumn{4}{|l|}{ Existe evidência de demanda reprimida no serviço? } \\
\hline \multicolumn{4}{|l|}{$\begin{array}{l}\text { Existe um sistema de marcação de consultas e sessões de fisioterapia de forma a organizar a } \\
\text { demanda? }\end{array}$} \\
\hline \multicolumn{4}{|l|}{ Existe espera para o usuário ter acesso à consulta? } \\
\hline \multicolumn{4}{|l|}{0 tempo entre a marcação da consulta e o início do tratamento é longo? } \\
\hline \multicolumn{4}{|l|}{ Existe um sistema de otimização do atendimento para reduzir ou evitar filas? } \\
\hline \multicolumn{4}{|l|}{ Existe o registro de alta ou encaminhamento de usuários para outras unidades ou serviços? } \\
\hline \multicolumn{4}{|l|}{0 serviço dispõe de indicadores para avaliação de capacidade instalada? } \\
\hline \multicolumn{4}{|l|}{0 serviço possui manuais de normas ou rotinas atualizados, disponíveis, de fácil acesso e em uso? } \\
\hline \multicolumn{4}{|l|}{ Existe flexibilidade de opção de horários das consultas ou sessões? } \\
\hline \multicolumn{4}{|l|}{$\begin{array}{l}0 \text { serviço gerencia o quantitativo de consultas e atendimentos, comparando o planejado com } 0 \\
\text { executado? }\end{array}$} \\
\hline \multicolumn{4}{|l|}{0 serviço dispõe de um fluxo de referência e contrarreferência dos usuários? } \\
\hline \multicolumn{4}{|l|}{$\begin{array}{l}0 \text { serviço utiliza métodos que permitem ao usuário identificar com facilidade os profissionais que } \\
\text { cuidam de sua saúde? }\end{array}$} \\
\hline \multicolumn{4}{|l|}{ Existem mecanismos que incorporam a Política Nacional de Humanização (PNH) em saúde no serviço? } \\
\hline \multicolumn{4}{|l|}{$\begin{array}{l}\text { Considerando os parâmetros assistenciais da Resolução n. 387/2011, percebe-se que a relação entre a } \\
\text { produtividade do serviço e a quantidade de profissionais disponíveis é equilibrada? }\end{array}$} \\
\hline \multicolumn{4}{|l|}{$\begin{array}{l}\text { Considerando os parâmetros assistências da Resolução n. 387/2011, percebe-se que a relação entre a } \\
\text { produtividade do serviço e a jornada de trabalho dos profissionais é equilibrada? }\end{array}$} \\
\hline \multicolumn{4}{|l|}{ Os serviços prestados estão vigentes na tabela do SUS? } \\
\hline \multicolumn{4}{|l|}{$\begin{array}{l}\text { Em caso de regime ambulatorial, o serviço respeita o limite de franquear até dez sessões/usuário a } \\
\text { cada nova reavaliação? }\end{array}$} \\
\hline \multicolumn{4}{|l|}{ As reavaliações acontecem pontualmente a cada dez sessões? } \\
\hline \multicolumn{4}{|l|}{$\begin{array}{l}\text { Em caso de regime hospitalar, o serviço respeita o quantitativo de sessões/dia admitidos na guia de } \\
\text { Autorização de Internação Hospitalar (AlH)? }\end{array}$} \\
\hline A cada reavaliação é gerado um relatório de avaliação funcional? & & & \\
\hline
\end{tabular}

\begin{tabular}{l|l|l|l}
\hline Bloco VI - VERIFICAÇÃO DOS PRONTUÁRIOS & Sim & Não & N / A \\
\hline & & & \\
\hline $\begin{array}{l}\text { Asserviço dispõe de instrumentos de registro ou prontuários de seus atendimentos? } \\
\text { mantêm organizados em local de fácil acessos? }\end{array}$ & & \\
\hline O registro dos prontuários é informatizado? & & & \\
\hline
\end{tabular}




\section{Bloco VI - VERIFICAÇÃO DOS PRONTUÁRIOS}

Os registros encontram-se legíveis, claros e coerentes?

Os registros foram feitos desde o primeiro contato do usuário com o serviço e são mantidos a cada intervenção?

0 prontuário dispõe de todos os itens necessários à identificação do usuário tais como: nome, idade, sexo, endereço, cor, etc.?

Existe evidência da história da doença atual do usuário, medicações em uso e tratamentos atuais?

Existe evidência de informações sobre antecedentes relevantes, histórico familiar, bem como tratamentos anteriores?

Existe evidência de informações sobre os hábitos de vida do usuário?

Existe descrição do exame físico, bem como instrumentos de avaliação utilizados, testes específicos, entre outros?

Os achados da avaliação no exame físico estão devidamente descritos?

Existe registro dos achados nos exames complementares?

0 prontuário contém o diagnóstico cinésico-funcional?

0 prontuário expressa um plano de intervenções com objetivos e condutas?

Os objetivos e condutas estão categorizados em curto, médio e longo prazo?

Existe evidência de evoluções no plano de tratamento?

As orientações dadas ao usuário são registradas?

Existe registro datado e evidência de realização das reavaliações dentro do prazo previsto?

Os relatórios de reavaliação funcional contêm informações sobre o quadro clínico inicial, período de tratamento, intervenções realizadas e resultados obtidos?

0 número de sessões prescritas foi especificado?

Existe evidência de alta programada?

Nos casos de conclusão do plano de intervenção e alta, são gerados relatórios?

Os procedimentos descritos e cobrados são compatíveis com os da tabela do SUS?

Existe evidência de cobrança indevida de procedimentos com códigos de maior valor?

Os procedimentos cobrados estão devidamente registrados no prontuário e condizentes com o plano de tratamento?

As codificações dos procedimentos cobrados estão sendo feitas corretamente?

0 registro de sessões usuário/dia é compatível com o número máximo estipulado na tabela unificada para serviços de fisioterapia em regime hospitalar?

0 registro de sessões usuário/mês é compatível com o número máximo estipulado na tabela unificada para serviços de fisioterapia em regime ambulatorial?

0 prontuário dispõe da assinatura e carimbo do profissional que realizou a avaliação ou a cada intervenção?

Existe evidência de rasura nas anotações bem como uso de corretor?

As folhas do registro são numeradas?

\begin{tabular}{l|l|l|l} 
Sim & Não & N / A \\
\hline & & \\
\hline & &
\end{tabular}

As tohas do registo sao numeradas?

\begin{tabular}{l|l|l|l}
\hline Bloco VII - VERIFICAÇÃO DA GESTÃO DE RESULTADOS & Sim & Não & N / A \\
\hline & & & \\
\hline 0 serviço dispõe de dados que comprovem o quantitativo de intervenções resolutivas? & & & \\
\hline 0 serviço avalia o desempenho de seus colaboradores? & & & \\
\hline 0 serviço avalia a satisfação de seus colaboradores? & & & \\
\hline 0 serviço estabelece suas metas e avalia o seu cumprimento? & & \\
\hline
\end{tabular}




\begin{tabular}{|c|c|c|c|}
\hline \multicolumn{4}{|l|}{ Bloco VII - VERIFICAÇÃO DA GESTÃO DE RESULTADOS } \\
\hline \multirow[b]{2}{*}{$\begin{array}{l}0 \text { serviço dispõe de meios de comunicação que permitam ao usuário expressar suas reclamações, } \\
\text { sugestôes e solicitações? }\end{array}$} & $\operatorname{Sim}$ & Não & $\mathrm{N} / \mathrm{A}$ \\
\hline & & & \\
\hline \multicolumn{4}{|l|}{ As reclamações e sugestões dos usuários são atendidas e em tempo hábil? } \\
\hline \multicolumn{4}{|c|}{$\begin{array}{l}0 \text { serviço realiza avaliações periódicas da satisfação de seus usuários utilizando seus resultados como } \\
\text { apoio decisório? }\end{array}$} \\
\hline \multicolumn{4}{|l|}{ Existe um controle estatístico da cobertura e acesso do serviço? } \\
\hline \multicolumn{4}{|c|}{$\begin{array}{l}\text { São realizadas reuniões periódicas para discussão das limitações e problemas do serviço, bem como } \\
\text { para propor soluções? }\end{array}$} \\
\hline \multicolumn{4}{|l|}{ Existe registro que comprove a ocorrência dessas reuniões? } \\
\hline \multicolumn{4}{|l|}{0 serviço utiliza indicadores para avaliação de seus resultados? } \\
\hline Os resultados dos indicadores são divulgados para melhoria na qualidade do serviço? & & & \\
\hline
\end{tabular}

\begin{tabular}{|c|c|c|c|}
\hline \multicolumn{4}{|l|}{ Bloco VIII - VERIFICAÇÃO DA SATISFAÇÃO DOS USUÁRIOS } \\
\hline 0 seu tratamento foi realizado por: & Sim & Não & $\mathrm{N} / \mathrm{A}$ \\
\hline \multicolumn{4}{|l|}{ Profissional fisioterapeuta? } \\
\hline \multicolumn{4}{|l|}{ Aluno ou estagiário? } \\
\hline \multicolumn{4}{|l|}{ Você teve dificuldade em marcar a consulta com o fisioterapeuta? } \\
\hline \multicolumn{4}{|l|}{ Existem filas de longa espera para se ter acesso ao serviço? } \\
\hline \multicolumn{4}{|l|}{ Após a consulta houve demora em iniciar as sessões de fisioterapia? } \\
\hline \multicolumn{4}{|l|}{ Os profissionais do serviço Ihe tratam com cortesia e respeito? } \\
\hline \multicolumn{4}{|c|}{$\begin{array}{l}\text { Os profissionais do serviço respeitaram a sua autonomia dando-lhe a oportunidade de expressar sua } \\
\text { opinião? }\end{array}$} \\
\hline \multicolumn{4}{|l|}{ Você teve oportunidade de escolher entre os horários das sessões? } \\
\hline \multicolumn{4}{|l|}{0 fisioterapeuta Ihe ouviu com atenção? } \\
\hline \multicolumn{4}{|l|}{0 fisioterapeuta Ihe explicou os objetivos e prazos do tratamento? } \\
\hline \multicolumn{4}{|l|}{0 fisioterapeuta responsável pelo tratamento informou o nome dele? } \\
\hline \multicolumn{4}{|l|}{ Os resultados da avaliação fisioterapêutica foram explicados? } \\
\hline \multicolumn{4}{|l|}{0 fisioterapeuta utilizou uma linguagem simples que permitiu sua compreensão? } \\
\hline \multicolumn{4}{|l|}{ Foram explicados os prós e contras das condutas do plano de intervenção? } \\
\hline \multicolumn{4}{|l|}{ Você sente ou sentiu segurança no atendimento? } \\
\hline \multicolumn{4}{|l|}{ Caso necessitasse novamente, utilizaria este serviço outra vez? } \\
\hline \multicolumn{4}{|l|}{ Os progressos do tratamento Ihe foram mostrados? } \\
\hline \multicolumn{4}{|l|}{ Foi avisado com antecedência sobre a data de sua alta? } \\
\hline \multicolumn{4}{|l|}{ Você foi chamado pelo seu nome? } \\
\hline \multicolumn{4}{|l|}{ Os profissionais do serviço se apresentaram falando seus nomes? } \\
\hline \multicolumn{4}{|l|}{ Você teve a opção de escolher entre vários tipos de tratamento? } \\
\hline \multicolumn{4}{|l|}{ Foi solicitado que você realizasse alguma atividade com a qual não concordava? } \\
\hline \multicolumn{4}{|l|}{ Você acha que o número de fisioterapeutas é suficiente para atender a este serviço? } \\
\hline \multicolumn{4}{|l|}{ Os resultados obtidos atenderam às suas expectativas? } \\
\hline \multicolumn{4}{|l|}{0 serviço se encontra em uma boa localização? } \\
\hline \multicolumn{4}{|l|}{ Você acha que 0 ambiente é agradável? } \\
\hline \multicolumn{4}{|l|}{ Houve necessidade de espera por conta da falta de algum equipamento ou material? } \\
\hline Você está satisfeito com a limpeza do ambiente? & & & \\
\hline
\end{tabular}




\begin{tabular}{l|l|l|l}
\hline Bloco VIII - VERIFICAÇÃO DA SATISFAÇÃO DOS USUÁRIOS & Sim & Não & N /A \\
\hline O seu tratamento foi realizado por: & & & \\
\hline Em geral, você diria que ficou satisfeito com o serviço? & & & \\
\hline Você gostou de ser atendido por este serviço de fisioterapia? & & \\
\hline
\end{tabular}

